\title{
Ueber Anzeichen von dem Vorhandensein eines Planeten, welcher (151) Abundantia in grosser Nähe begleitet.
}

\author{
Von $v . d$. Groeben, Oberstlieutenant a. D.
}

Die Bearbeitung des Planeten (15I) Abundantia ist schon Anfangs der achtziger Jahre auf besondere Schwierig. keiten gestossen, welche bereits damals zu der Annahme führten, es sei in einer der ersten Oppositionen nach dem Entdeckungsjahr ein noch unbekannter, zufällig nahe an gleicher Himmelsstelle erschienener Planet irrthümlich für den gesuchten gehalten worden. Eine jetzt vorgenommene gründliche Discussion des gesammten vorhandenen Beobachtungsmaterials hat diese Annahme der Art bestätigt, dass Zweifel an ihrer Realität kaum mehr möglich sind. Aber noch Auffälligeres hat sich hierbei herausgestellt. Es gelang nämlich zwar Bahnelemente zu ermitteln, welche 5 Normalörter - 4 Oppositionen angehörend und einen Zeitraum von 12 Jahren umfassend - mit einer, auch strenge Anforderungen völlig befriedigenden Genauigkeit darstellen; dagegen ergaben sich beim Vergleich der Beobachtungen aus 3 andern Oppositionen mit der Rechnung nach jenen Elementen ganz erhebliche Differenzen; und in allen 3 Fällen hatten die, an Grösse nicht sehr verschiedenen $A b$ weichungen gleiches Vorzeichen.

Unter solchen Umständen ist die Voraussetzung, dass es sich bei den betreffenden Beobachtungen jedesmal um einen andern, noch unbekannten Planeten gehandelt haben könne, ohne $Z$ weifel so unwahrscheinlich, dass sie ernstlich nicht in Betracht kommen kann. Eher dürfte die Erklärung Glauben verdienen, dass die Widersprüche auf die Existenz eines Begleiters zurückzuführen seien, nahe genug, um gelegentlich mit Abundantia verwechselt werden zu können, und doch weit genug $a b$, um das gleichzeitige Erscheinen beider Gestirne im Gesichtsfelde des Fernrohrs auszuschliessen. Denn, neigt man der Hypothese zu, dass die Asteroiden die Reste eines, in Urzeiten durch eine Katastrophe zersprengten grösseren Planeten seien, so wird man auch die Möglichkeit nicht unbedingt in Abrede stellen können, dass damals unmittelbar zusammenliegende Theile des Gesammtkörpers ausnahmsweise auch nahe gleichen Impuls empfangen haben könnten.

Am nächsten pflegt freilich unter ähnlichen Umständen die Vermuthung zu liegen, dass die entstandenen Schwierigkeiten einfach das Ergebniss von Rechenfehlern seien. Hiergegen sprechen indessen im vorliegenden Falle: das Maass der peinlichen Vorsicht, womit die Rechnungen ausgefuhrt sind, so wie die Vielseitigkeit und Strenge der, wo es irgend möglich war, angewendeten Proben. Die nachfolgende Skizze des Ganges der Untersuchung mag dies näher begründen.

Die hier mitgetheilten Abundantia-Elemente basiren auf 5 neu hergeleiteten Normalörtern, von denen 2 dem Entdeckungsjahr I875, die übrigen den Jahren I879, 86 und 87 angehören. Die drei ersten (von 1875 und 79) haben, sowohl wegen der Zahl der benutzten Beobachtungen, als auch wegen der guten Uebereinstimmung derselben unter sich, begründeten Anspruch auf vollste Zuverlässigkeit. Die beiden letzten (von I 886 und 87 ) bieten weniger Sicherheit, weil zu ihrer Ableitung nur je zwei Beobachtungen vorhanden waren, welche uberdies beim Vergleich mit der Ephemeride unter einander, namentlich in Declination, in beiden Fällen ziemlich stark differirten.

Die Störungen durch Jupiter und Saturn sind für den ganzen Zeitraum von 1875 bis 1896 mit den zur Verfügung stehenden besten Elementen nach der Methode der Variation der Constanten neu berechnet; die Jupiterstörungen 5 stellig, die Saturnstörungen 4 stellig. Ein Wechsel der Elemente durch Anrechnung der inzwischen angelaufenen Störungen hat hierbei im Allgemeinen nach jedem 18 . der 40 tägigen Intervalle, insoweit sich aber die Jupiternähe fühlbar machte, nach jedem 9., stattgefunden. Später sind dann für die beiden Zeiträume von 1877 Aug. 1 2.0 bis 1879 Oct. 21.0 und von 1890 Oct. 3.0 bis 1892 Dec. I I.0 die StörungsRechnungen völlig streng nach der Methode der polaren Coordinaten, für Jupiter 6 stellig, für Saturn 5 stellig, noch einmal wiederholt, um bezüglich der Wirkung der beiden, gegen Ende der Jahre 1878 und $189 \mathrm{I}$ eingetretenen Jupiternähen völlige Sicherheit zu erhalten. In beiden Fällen waren die Abweichungen zwischen den Resultaten der genäherten und der strengen Rechnung nicht grösser, als nach Lage der Dinge von vorn herein erwartet werden musste. Die Fehlerlosigkeit beider Rechnungen ist hierdurch für die genannten Zeiträume ausser Zweifel gestellt. Die Richtigkeit der übrigen Theile der Störungsrechnung ist durch den genau controllirten, regelmässigen Gang der Differenzen verbürgt, einschliesslich für eine dritte, auf die Mitte des Jahres I 885 fallende Jupiternähe, bei welcher die Störungen nicht entfernt die Höhe, wie in den Jahren 1878 und 91 erreichten.

Der erste, leidlich befriedigende Bahnverbesserungsversuch auf Grund der oben erwähnten 5 Normalörter führte zur folgenden Darstellung der letzteren:

$$
\begin{array}{clll}
\text { Normalort } 1875 \mathrm{I} & \mathrm{d} G=+2 " \mathrm{I} & \mathrm{d} g=-0.4 \\
* & 1875 \mathrm{II} & =+0.3 & =-1.6 \\
* & 1879 & =-5.2 & =-3.6 \\
* & 1886 & =+0.9 & =-3.7 \\
* & 1887 & =+1.6 & =+2.9
\end{array}
$$

Der, hiernach namentlich in den Werthen von $\mathrm{d} G$ deutlich hervortretende ${ }_{\text {Gang }}$, bei welchem die grösste, negative Abweichung ( -5.2 ) gerade auf den ganz besonders gut verbürgten Normalort für I 879 entfiel, gab Veranlassung zu der Vermuthung, dass vielleicht die Unsicherheit der genäherten Störungsrechnung während der Jupiternähe 1878 die Erzielung eines bessern Resultats verhindert haben möge. Der Bahnverbesserungsversuch wurde daher nach strenger Wiederholung jenes Theils der Störungsrechnung noch ein. mal durchgeführt und ergab nun folgende Endabweichungen : 


$$
\text { Normalort } \begin{array}{rlr}
\text { 1875 I } \mathrm{d} G=+0.6 & \mathrm{~d} g=0.0 \\
1875 \mathrm{II} & =-1.5 & =-0.2 \\
1879 & =+2.3 & =-3.7 \\
1886 & =+0.2 & =-2.2 \\
\text { 1887 } & =-0.8 & =+2.5
\end{array}
$$

Ein Resultat, wie es besser, in Betracht der Unsicherheit der Declinationen der Normalörter für 1886 und 87 , gewiss nicht erwartet werden konnte.

Bei beiden Versuchen sind die Restfehler der Bedingungsgleichungen, welche durch Einsetzen der Bahnverbesserungswerthe in jene Gleichungen gefunden wurden, mit den Resultaten der Rückrechnung der Normalörter mittelst der verbesserten Elemente verglichen worden. Die hierdurch ermittelten Differenzen der correspondirenden Fehlbeträge beschränkten sich durchweg auf geringe Bruchtheile der Bogensecunde, blieben also völlig in den Grenzen der unvermeidlichen Unsicherheit der zur Anwendung gekommenen 6 stelligen logarithmischen Rechnung. Auch die Richtigkeit der Bahnverbesserungs-Rechnung unterliegt hiernach keinem Zweifel.

Nach so günstigem Resultat hatte die Erwartung volle Berechtigung, dass die Rechnung mit den verbesserten Elementen auch die sonst noch bekannt gewordenen Beobachtungen des Planeten wenigstens mit annähernder Genauigkeit darstellen müsse. Dies ist nun aber keineswegs der Fall.

Weitere Beobachtungen von (I5I) Abundantia sind publicirt aus den Jahren I 88 I, 85 und 94. Dementsprechend sind 3 weitere Normalörter gebildet worden.

Der für $188 \mathrm{I}$ ist abgeleitet aus 5, nur die 3 Tage von Februar 22 bis 24 umfassenden Beobachtungen der 3 Sternwarten: Düsseldorf, Königsberg und Leipzig. Die Abweichung von der, im Berliner Jahrbuch für I 883 nach Tietjen'schen Elementen publicirten Ephemeride war ziemlich bedeutend. Sie betrug im arithmetischen Mittel:

$$
\mathrm{d} \alpha=+22.45 ; \mathrm{d} \delta=-55^{\prime \prime} \cdot 3,
$$

im Sinne B - R. Die Einzelwerthe der Abweichungen stimmten unter einander, wenn auch nicht gerade besonders genau, so doch immerhin befriedigend überein.

Der Normalort für 1885 beruht auf 2 Wiener Beob. achtungen, die beim Vergleich mit der Ephemeride unter einander in $\alpha$ eine Differenz von 0.42 zeigten. Dies gab Veranlassung zu dem Entschluss, den Ort, unbeschadet seiner Verwendung zur Controlle, von den BahnverbesserungsVersuchen auszuschliessen.

Endlich sind Beobachtungen von (151) Abundantia für 1894 April 7 und April 25 zu Algier im Bulletin astronomique und in den Astr. Nachr, veröffentlicht. Aus erstgenannter Publication (Bd. I I, S. 349) geht hervor, dass die 4 daselbst specialisirten Beobachtungen von April 7 die Resultate der Ausmessung zweier photographischer Platten durch je 2 Beobachter darstellen. Sie sind alle 4 auf ein und denselben Vergleichstern des Weisse-Catalogs bezogen. Für April 25 sind 2 Positionen angegeben, welche durch directe Beobachtung gewonnen sind. Der Vergleich aller dieser Beobachtungen mit einer ad hoc berechneten Ephemeride ergab das sehr auffällige Resultat, dass die Differenzen zwischen Beobachtung und Rechnung, obwohl an sich sehr bedeutend (über $24^{\text {s }}$ in $\alpha$ und über $3^{\prime}$ in $\delta$ ), doch für die
Zeit vom 7. bis 25. April einen verhältnissmässig nur ganz geringen Gang zeigten. Dieselbe betrug in $\alpha$ wenig über eine Zeitsecunde, in $\delta$ nur wenige Bogensecunden. Dabei stimmten die vier Beobachtungen von April 7 unter einander ganz befriedigend überein und wurden daher zu einem neuen Normalort vereinigt, um auch ihrerseits zu Bahnverbesserungs-Versuchen, oder mindestens zur Controlle verwendet werden zu können.

Alle diese drei neuen Normalörter fügen sich der Rechnung mit den verbesserten Elementen nicht. Die Rückrechnung nach Ausführung der Bahnverbesserung ergab neben der oben mitgetheilten, sehr befriedigenden Darstellung der angeschlossenen fünf Normalörter, für die nicht angeschlossenen von $188 \mathrm{r}$ und 94 die bedeutenden Fehlbeträge von $\mathrm{d} G=+6^{\prime} 12^{\prime \prime} 7$ respective $+8^{\prime} 4^{\prime \prime} .7$. In guter Uebereinstimmung hiermit lieferten die betreffenden $\mathrm{Be}$ dingungsgleichungen beim Einsetzen der BahnverbesserungsWerthe in dieselben die correspondirenden Beträge:

$$
\mathrm{d} G=+6^{\prime} \text { 1 3."6 und +8'47"0. }
$$

Zur weiteren Controlle sind dann später mit den verbesserten Elementen auch noch die Aequator-Coordinaten für die Epochen aller drei nicht angeschlossenen Oerter nachgerechnet und die Resultate mit den Normalörtern selbst verglichen worden. Hierbei ergaben sich im Sinne $B-R$ :

$$
\begin{aligned}
& \text { Normalort } 188 \mathrm{r} \text { d } \alpha=+25^{5} .29 \text { d } \delta=-2^{\prime} \mathrm{r} 9 \text { ". } \mathrm{r} \\
& { }^{\circ} 1885=+38.20=-2 \quad 0.8 \\
& \triangle 1894=+30.48=-355.3
\end{aligned}
$$

Alles weitere Bemühen, die Ursachen dieser Differenzen im Rechnungswege zu ermitteln, ist vergeblich gewesen. Die ausgeführten Versuche, auch nur einen der Normalörter für I $88 \mathrm{I}$, oder 1894 als vierten, lediglich mit den beiden für I 875 und den für 1879 , behufs Herleitung einer verbesserten Bahn zu combiniren, sind gänzlich fehlgeschlagen. Und doch sind nicht nur die Oerter für 1875 völlig einwandfrei. Der für 1879 ist es nicht minder, weil er aus vier, den Zeitraum von September 23 bis November 18 umfassenden, Berliner Beobachtungen hergeleitet ist, welche beim Vergleich mit der Ephemeride eine ganz ungewöhnlich genaue Uebereinstimmung unter einander gezeigt haben. Nur im Wege weiterer Beobachtung wird daher über die noch ungelösten Widersprüche und insbesondere auch daruber Klarheit erlangt werden können, ob, wie diesseits vermuthet wird, die seither auf Abundantia allein bezogenen Beobachtungen in der That zwei verschiedenen Planeten zugehören, welche in geringer Entfernung von einander sich in nahezu gleichen Bahnen bewegen. Dass die Bestätigung dieser Vermuthung ganz dazu geeignet sein würde, den Ausgangspunkt weiterer, allgemein interessanten Untersuchungen und Schlussfolgen zu bilden, liegt auf der Hand. Vielleicht geben daher gegenwärtige Darlegungen die Anregung dazu, dass die photographischen Platten, welche der Publication der $1894^{\mathrm{er}}$ Beobachtungen zu Algier als Grundlage gedient haben, sofern sie noch erhalten sein sollten, mit Bezug auf den fraglichen Punkt einer erneuten Prüfung unterworfen werden. Jedenfalls aber wird die, im November dieses Jahres bevorstehende, recht günstig liegende Opposition von Abundantia Gelegenheit bieten, die einschlägigen 
Beobachtungen aufzunehmen. Es ergeht daher hiermit an diejenigen Sternwarten, in deren Arbeitsprogramm die gelegentliche Beobachtung kleiner Planeten eine Stelle findet, die ganz ergebenste Bitte:

1) nach der hier beigegebenen Ephemeride (berechnet mittelst der ebenfalls mitgetheilten Elemente) Abundantia zu beobachten, und

2) nach Anbringung der oben angegebenen aequatorealen Abweichungen der Normalörter für 1881, 85 und 94 als Correctionen der Ephemeride, in den hierdurch gegebenen, ungefähren Grenzen auch nach dem vermutheten Begleiter zu suchen.

Besonders mühevoll dürfte sich Beides, selbst wenn man von dem, hier gewiss bequemen, photographischen Verfahren absieht, kaum gestalten, da nach Erfahrungen analoger Fälle der wirkliche Ort des Planeten von dem vorausberechneten unter den obwaltenden Umständen kaum um mehr als höchstens zwei bis drei Zeitsecunden verschieden erwartet werden darf und da ferner die Helligkeit des, doch nur auf ziemlich eng begrenztem Raum zu suchenden Begleiters mindestens als gleichwerthig mit der von Abundantia vorauszusetzen ist, - weil anders die vermutheten Verwechselungen kaum möglich wären.

Elemente des Planeten ( 151 ) Abundantia.

Epoche und Osculation I 896 Nov. 20.0 M. Z. Berlin.

$$
\begin{aligned}
& M=254^{\circ} 35^{\prime} 51^{\prime \prime} .5 \\
& \omega=1313222.5 \\
& \delta=3^{8} 543^{8.1} 1900.0 \\
& i=62818.5 \\
& \varphi=2 \text { Io } 15.4 \\
& \mu=850.8499 \\
& \log a=0.413435^{8}
\end{aligned}
$$

\begin{tabular}{|c|c|c|c|c|c|c|c|}
\hline 1896 & \multicolumn{2}{|c|}{$\alpha$ app. } & \multicolumn{3}{|c|}{$\delta$ app. } & $\log \Delta$ & Ab. $\cdot Z$ \\
\hline Nov. 22 & \multicolumn{2}{|c|}{$3^{\mathrm{h}} 53^{\mathrm{m}} 3^{\mathrm{s}} \cdot 26$} & \multicolumn{3}{|c|}{$+24^{\circ} 20^{\circ} 4^{8.7}$} & 0.213194 & $13^{\mathrm{m}} 33^{\mathrm{s}}$ \\
\hline 23 & 52 & $27 \cdot 72$ & 24 & 20 & 0.0 & 213175 & 33 \\
\hline 24 & $5 I$ & 23.27 & 24 & 19 & $7 \cdot 3$ & 213232 & 33 \\
\hline 25 & $5^{\circ}$ & 19.00 & 24 & 18 & 10.6 & 213366 & 34 \\
\hline 26 & 49 & 14.99 & 24 & 17 & 10.0 & 213577 & 34 \\
\hline 27 & $4^{8}$ & I I.3 I & 24 & 16 & 6.0 & 213864 & 35 \\
\hline 28 & 47 & 8.05 & 24 & 14 & 58.6 & 214228 & 35 \\
\hline 29 & 46 & 5.28 & 24 & 13 & 48.1 & 214667 & 36 \\
\hline 30 & 45 & 3.09 & 24 & 12 & 34.8 & 215182 & 37 \\
\hline Dec. I & 44 & J.55 & 24 & I I & I 8.9 & $21577 \mathrm{I}$ & 38 \\
\hline 2 & 43 & 0.73 & 24 & 10 & 0.7 & 216434 & 39 \\
\hline 3 & 42 & 0.72 & 24 & 8 & 40.4 & 217170 & 41 \\
\hline 4 & $4 !$ & 1.59 & 24 & 7 & 18.3 & 217979 & 42 \\
\hline 5 & 40 & $3.4 \mathrm{I}$ & 24 & 5 & 546 & 218859 & 44 \\
\hline 6 & 39 & 6.24 & 24 & 4 & 29.6 & 219809 & 46 \\
\hline 7 & $3^{8}$ & IO.I 7 & 24 & 3 & 3.6 & 220828 & 48 \\
\hline 8 & 37 & I 5.25 & 24 & $\mathbf{I}$ & $3^{6.8}$ & 221916 & 50 \\
\hline 9 & $3^{6}$ & $21 \cdot 5^{6}$ & 24 & o & 9.5 & 223070 & 52 \\
\hline 10 & 335 & 29.16 & +23 & $5^{8}$ & 42.0 & 0.224290 & 1354 \\
\hline
\end{tabular}

Ephemeride für I $^{\text {h }}$ M. Z. Berlin.

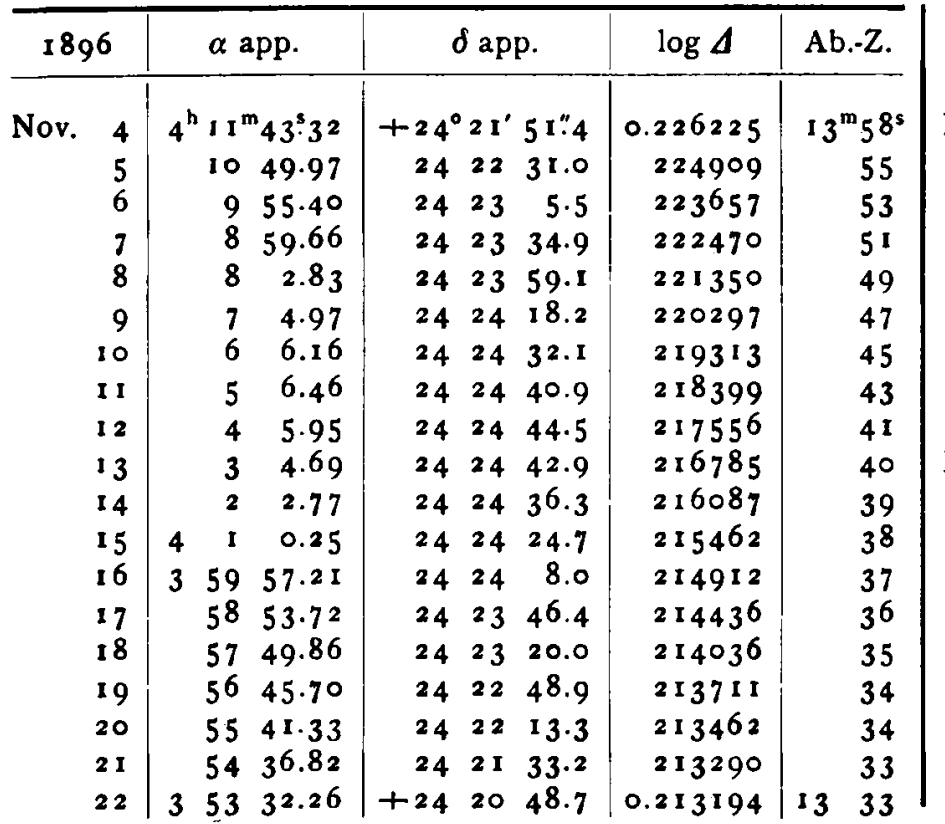

Opp. in AR. Nov. $2 \mathrm{I}$.

$5(\log \Delta+\log r)=3.155$.

Grösse $=11.8$.

Berlin im Juni 1896 .

v. d. Groeben, Oberstlieutenant a. D.

Beobachtungen von veränderlichen Sternen.

Von G. Gruss.

Ch. 906, R Trianguli.

Wurde vom I. Januar bis zum I 7. März $1896 \quad 13 \mathrm{mal}$ beobachtet; der Stern nahm stetig ab bis zum 7. März, zu welcher Zeit er sich an der Grenze der Sichtbarkeit für den Achtzöller von Reinfelder \& Hertel befand; am I 7. März war der Stern I r. Grösse.

\section{Ch. ${ }^{367}, \mathrm{X}$ Tauri.}

Die I 2 Vergleichungen dieses Sterns bei durchgehends guter, durchsichtiger Luft, die sich auf den Zeitraum vom 24. Januar bis 17. März 1896 erstrecken, deuten an, dass die Periode des Lichtwechsels sehr kurz (etwa 2.9 Tage) ist oder dass der Stern sein Licht unregelmässig ändert.

\section{Ch. 1577, R Tauri.}

Wurde vom 10. Januar bis I 7 . März I 896 I 8 mal beobachtet. Der Stern war Anfangs $101 / 2$ ter Grösse, wuchs hierauf ziemlich schnell und erreichte sein Maximum 8.6 Grösse am 7. Februar; dann nahm der Stern langsam ab bis zum 7. März $\left(9^{\mathrm{m}} \cdot 3\right)$; am letzten Beobachtungstage 17 . März war der Stern 10. Grösse. 